\title{
Convergence of the Quantum Boltzmann Map
}

\author{
R. F. Streater * \\ Bedford College, Regent's Park, London, NW1 5NS, England
}

\begin{abstract}
We consider a non-linear map on the space of density matrices, which we call the Boltzmann map $\tau$. It is the composition of a doubly stochastic map $T$ on the space of $n$-body states, and the conditional expectation onto the one-body space. When $T$ is ergodic, then the iterates of $\tau$ take any initial state to the uniform distribution. If the energy levels are equally spaced, and $T$ conserves energy and is ergodic on each energy shell, then iterates of $\tau$ take any initial state of finite energy to a canonical distribution.
\end{abstract}

\section{Introduction}

(1.1) This paper is the quantum version of [1]. Let $\mathscr{H}$ be a Hilbert space with $\operatorname{dim} \mathscr{H}=N \leqq \infty$. A (normal) state $\varrho$ is then a positive operator with unit trace. We denote the set of trace-class operators by $\mathscr{B}(\mathscr{H})_{1}$ and the normal ${ }^{1}$ states by $\sigma(\mathscr{H})$. A stochastic map is a linear map $T$ from $\mathscr{B}(\mathscr{H})$ to $\mathscr{B}(\mathscr{H})$ mapping $\sigma(\mathscr{H})$ to itself and preserving the trace: $\operatorname{Tr}(T \varrho)=\operatorname{Tr} \varrho, \varrho \in \mathscr{B}(\mathscr{H})_{1}$. A doubly stochastic map is a stochastic map $T$ such that $T 1_{N}=1_{N}$, where $1_{N}$ is the identity on $\mathscr{H}$ [4].

A unitary or anti-unitary conjugation $\varrho \mapsto T \varrho=U \varrho U^{-1}$ is doubly stochastic, as is any convex combination of such maps.

(1.2) Let $\mathscr{K}$ be a Hilbert space, the one-particle space, and

(1.3) let $\mathscr{H}=\mathscr{K} \otimes \ldots \otimes \mathscr{K}$ ( $n$ factors) be the $n$-particle space.

We shall be interested in a doubly stochastic map $T: \mathscr{B}(\mathscr{H}) \rightarrow \mathscr{B}(\mathscr{H})$ that preserves the symmetry under permutations of the factors $\mathscr{K}$. To such a $T$ we define the corresponding Boltzmann map $\tau$ to be the composition of maps:

$$
\varrho \mapsto \varrho \otimes \ldots \otimes \varrho \mapsto T(\varrho \otimes \ldots \otimes \varrho) \mapsto \operatorname{Tr}_{2 \ldots n} T(\varrho \otimes \ldots \otimes \varrho)=\tau(\varrho) .
$$

Here, $\operatorname{Tr}_{2 \ldots n}$ means the trace over the second, third, .., $n^{\text {th }}$ factors $\mathscr{K}$. Obviously, (1.4) defines a non-linear map $\tau: \sigma(\mathscr{K}) \rightarrow \sigma(\mathscr{K})$.

* Present address: Department of Mathematics, Kings College Strand, London WC2 R2LS, England

1 Normal in the sense [2] of linear functionals on the $W^{*}$-algebra $\mathscr{B}(\mathscr{H})$, not in the sense of [3] 
(1.5) Alicki and Messer [5] have suggested a similar map for continuous time, where the analogue of $T$ is completely positive. Our choice is motivated by the following result:

(1.6) Theorem. Let $\varrho \in \sigma(\mathscr{K})$ have finite entropy: $S(\varrho)=-\operatorname{Tr} \varrho \log \varrho<\infty$. Then

$$
S(\tau \varrho) \geqq S(\varrho) \text {. }
$$

Proof.

$$
n S(\tau \varrho)=\sum_{j} S\left(\operatorname{Tr}_{1 \ldots \hat{j} \ldots n} T(\varrho \otimes \ldots \otimes \varrho)\right)
$$

by symmetry, where $\hat{j}$ means $j$ is omitted

$$
\geqq S(T(\varrho \otimes \ldots \otimes \varrho))
$$

by [6, Proposition 2.5.6]

$$
\geqq S(\varrho \otimes \ldots \otimes \varrho)
$$

by [4, Lemma 2-5, Corollary]

$$
=n S(\varrho) \text {. }
$$

(1.8) To show that $\tau^{m} \varrho$ converges to the uniform distribution if $N<\infty$, we must postulate some ergodic properties. Now, $T$ is a linear operator on the Hilbert space of Hilbert-Schmidt operators on $\mathscr{H}$. Let us say $T$ is ergodic if $1_{N}$ is the only fixedpoint of $T$ in $\mathscr{B}(\mathscr{H})$. Let us say that $T$ has a spectral gap $\Delta, 0<\Delta<1$ if it is ergodic and the spectrum of $T^{*} T$ is contained in $[0,1-\Delta] \cup\{1\}$.

\section{Entropy Gain Under a Doubly Stochastic Map}

We give a sharp estimate which will imply the convergence of $\tau^{m} \varrho$ when $T$ is ergodic.

(2.1) Lemma. Let $\mathscr{H}$ be a Hilbert space with $\operatorname{dim} \mathscr{H}=N<\infty$, and denote by $\mathscr{B}(\mathscr{H})_{2}$ the Hilbert space of operators on $\mathscr{H}$ with scalar product $\langle A, B\rangle=\operatorname{Tr}\left(A^{*} B\right)$. Let $T: \mathscr{B}(\mathscr{H})_{2} \rightarrow \mathscr{B}(\mathscr{H})_{2}$ be a doubly stochastic map, ergodic with spectral gap $\Delta$. Let $A \in \sigma(\mathscr{H})$ and let $B=T A$. Then

$$
S(B)-S(A) \geqq \frac{\Delta}{2}\left\|A-N^{-1} 1_{N}\right\|_{2}^{2} .
$$

Proof. Let $\left\{\varphi_{i}, a_{i}\right\}$ and $\left\{\psi_{i}, b_{j}\right\}$ be the orthonormal eigenvectors and eigenvalues of $A$ and $B$, respectively. Then $0 \leqq a_{i}, b_{j} \leqq 1$. Let $f(x)=x \log x, c_{i j}=\left\langle\varphi_{i}, \psi_{j}\right\rangle_{\mathscr{H}}$. Then, as in $[6,2.5 .2]$ we have

$$
\begin{aligned}
& \left\langle\varphi_{i},\left\{f(A)-f(B)-(A-B) f^{\prime}(B)-\frac{1}{2}(A-B)^{2}\right\} \varphi_{i}\right\rangle_{\mathscr{H}} \\
& \quad=\sum_{i, j}\left|c_{i j}\right|^{2}\left\{f\left(a_{i}\right)-f\left(b_{j}\right)-\left(a_{i}-b_{j}\right) f^{\prime}\left(b_{j}\right)-\frac{1}{2}\left(a_{i}-b_{j}\right)^{2}\right\} .
\end{aligned}
$$

Now, in the range of $a_{i}, b_{j}$ we have

$$
f(x)-f(y)-(x-y) f^{\prime}(y)=\frac{1}{2}(x-y)^{2} f^{\prime \prime}(\xi),
$$


where $0 \leqq \xi \leqq 1$ and $f^{\prime \prime}(\xi)=\frac{1}{\xi} \geqq 1$. Thus

$$
f\left(a_{i}\right)-f\left(b_{j}\right)-\left(a_{i}-b_{j}\right) f^{\prime}\left(b_{j}\right)-\frac{1}{2}\left(a_{i}-b_{j}\right)^{2} \geqq 0 .
$$

Summing over $i$ gives the following sharper form of [6, Proposition 2.5.3]:

i.e.

$$
\operatorname{Tr}\left\{A \log A-B \log B-(A-B)(\log B+1)-\frac{1}{2}(A-B)^{2}\right\} \geqq 0,
$$

$$
\operatorname{Tr}\{A(\log A-\log B)\} \geqq \frac{1}{2} \operatorname{Tr}(A-B)^{2} .
$$

By [4, Theorem 2-2], there exist unitaries $U_{\alpha}$ and non-negative numbers $w_{\alpha}$ with $\sum_{\alpha} w_{\alpha}=1$ and $B=T A=\sum_{\alpha} w_{\alpha} A_{\alpha}, A_{\alpha}=U_{\alpha} A U_{\alpha}^{-1}$. Then for each $\alpha$, $\operatorname{Tr} A_{\alpha}\left(\log A_{\alpha}-\log B\right) \geqq \frac{1}{2} \operatorname{Tr}\left(A_{\alpha}-B\right)^{2}$, so multiplying by $w_{\alpha}$ and summing, and noting that $\operatorname{Tr} A_{\alpha} \log A_{\alpha}=\operatorname{Tr} A \log A$ and $\sum_{\alpha} w_{\alpha}=1$ :

i.e.

$$
\operatorname{Tr}(A \log A-B \log B) \geqq \frac{1}{2} \sum_{\alpha} w_{\alpha} \operatorname{Tr}\left(A_{\alpha}-B\right)^{2}
$$

$$
\begin{aligned}
& S(B)-S(A) \geqq \frac{1}{2} \sum_{\alpha} w_{\alpha}\left\{\left\langle A_{\alpha}, A_{\alpha}\right\rangle-\left\langle A_{\alpha}, B\right\rangle-\left\langle B, A_{\alpha}\right\rangle+\langle B, B\rangle\right\} \\
& =\frac{1}{2}\{\langle A, A\rangle-\langle B, B\rangle\}=\frac{1}{2}\left\{\langle A, A\rangle-\left\langle A, T^{*} T A\right\rangle\right\} .
\end{aligned}
$$

Now $1_{N}$ is a simple eigenvalue of $T^{*} T$, and we may write the orthogonal decomposition $A=\frac{1}{N} 1_{N} \oplus\left(A-\frac{1}{N} 1_{N}\right)$. Hence

$$
\begin{aligned}
S(B)-S(A) & \geqq \frac{1}{2}\left\langle A,\left(1_{N}-T^{*} T\right) A\right\rangle \\
& =2^{-1}\left\langle A-N^{-1} 1_{N},\left(1_{N}-T^{*} T\right)\left(A-N^{-1} 1_{N}\right)\right\rangle \\
& \geqq \frac{\Delta}{2}\left\|A-\frac{1}{N} 1_{N}\right\|_{2}^{2}
\end{aligned}
$$

since $\Delta$ is the smallest eigenvalue of $1-T^{*} T$ apart from 0 .

(2.4) Corollary. Let $A=\varrho_{12}$ on $H_{1} \otimes H_{2}$, and $B=\varrho_{1} \otimes \varrho_{2}$, where $\varrho_{1}=\operatorname{Tr}_{2} \varrho_{12}$, etc. Then $-\operatorname{Tr} A \log A=S_{12},-\operatorname{Tr} A \log B=S_{1}+S_{2}$ in the sub-additive entropy inequality [4, Proposition 2.5.6] gives a quantitative estimate

$$
S_{1}+S_{2}-S_{12} \geqq \frac{1}{2}\left\|\varrho_{12}-\varrho_{1} \otimes \varrho_{2}\right\|_{2}^{2} .
$$

(2.5) Theorem. The microcanonical limit. Let $\operatorname{dim} K=k<\infty$, and $T$ a symmetry preserving ergodic doubly stochastic map on $K \otimes \ldots \otimes K$. Then for any $\varrho \in \sigma(K)$, $\tau^{m} \varrho \rightarrow k^{-1} 1_{K}$ as $m \rightarrow \infty$.

Proof. The entropy $S\left(\tau^{m} \varrho\right)$ is increasing and bounded above, and so converges. Hence the increment $S\left(\tau^{m+1} \varrho\right)-S\left(\tau^{m} \varrho\right)$ converges to 0 . In finite dimensions $\Delta>0$, so (2.2) implies that

and so $\tau^{m} \varrho \rightarrow k^{-1} 1_{\mathscr{K}}$, as $m \rightarrow \infty$.

$$
\left\|\tau^{m} \varrho \otimes \ldots \otimes \tau^{m} \varrho-\bigotimes_{1}^{n} k^{-1} 1_{\mathscr{K}}\right\|_{2} \rightarrow 0,
$$




\section{Energy Conservation}

(3.0) In order to discuss the canonical Gibbs state, we must introduce an energy operator $H$ on $\mathscr{K}$ ( $\mathscr{K}$ can be $\infty$-dimensional in what follows). Thus let $H$ have spectrum $0,1,2, \ldots$ and suppose that the multiplicity $m(j)$ of the energy-level $j$ is finite and that for some $\kappa>0$ and integer $r$,

$$
m(j) \leqq \kappa j^{r}, \quad j=1,2, \ldots .
$$

These conditions ensure that $e^{-\beta H}, \beta>0$, is of trace class. The equal spacing of the energy levels limits the theory to a rather special class; but it does allow thorough mixing to take place by scattering that conserves energy. This would not be possible if, for example, the energy levels were not commensurate.

(3.2) Let $H=\sum_{M}^{\infty} j\left(E_{j}-E_{j-1}\right)$ be the spectral resolution of $H$, and let $H_{M}=\sum_{1}^{M} j\left(E_{j}-E_{j-1}\right)$. Then $H_{M} \in \mathscr{B}(\mathscr{K})$. We say that a state $\varrho$ on $\mathscr{B}(\mathscr{K})$, not necessarily normal, has finite mean energy $\mathscr{E}$ if

$$
\lim _{M \rightarrow \infty} \operatorname{Tr}\left(\varrho H_{M}\right)=\mathscr{E}<\infty .
$$

(3.3) We again consider a doubly stochastic map $T$ on $\bigotimes^{n} \mathscr{K}=\mathscr{H}$. We require $T$ to mix up states in $\mathscr{H}$ of the same energy, but not to mix up states of differing energy. Thus let $\mathfrak{h}$ be the generator of $\bigotimes^{n} e^{i H t}$; then $\mathfrak{h}$ is an operator on $\mathscr{H}$ with spectrum $0,1,2, \ldots$ and having finite multiplicity. Let $\mathscr{H}_{\eta}, \eta=0,1, \ldots$ be the subspace with energy $\eta . \mathscr{B}\left(\mathscr{H}_{\eta}\right)$, called the "energy-shell $\eta$ " is a finite-dimensional space that can be identified with the subspace of $\mathscr{B}(\mathscr{H})_{2}$ consisting of operators mapping $\mathscr{H}_{\eta}$ to $\mathscr{H}_{\eta}$ and being zero on $\mathscr{H}_{\eta}^{\perp}$. In the scalar product of $(2.1)$, we may write $\mathscr{B}(\mathscr{H})_{2}$ as a direct sum of orthogonal subspaces

$$
\mathscr{B}(\mathscr{H})_{2}=\bigotimes_{\eta=0}^{\infty} \mathscr{B}\left(\mathscr{H}_{\eta}\right) \otimes \mathscr{L},
$$

where $\mathscr{L}$ is orthogonal to all energy shells. We consider doubly stochastic maps $T$ that mix up each energy shell $\mathscr{B}\left(\mathscr{H}_{\eta}\right)$ :

(3.4) $T$ maps $\mathscr{B}\left(\mathscr{H}_{\eta}\right)$ to itself, $\eta=0,1,2, \ldots$ and maps $\mathscr{L}$ to itself. Restricted to $\mathscr{B}\left(\mathscr{H}_{\eta}\right), T$ is ergodic. $T$ commutes with permutations of the $n$ factors $\mathscr{B}(\mathscr{K})$.

This class of doubly stochastic maps is the quantum analogue of the classical version [1]. Our assumption (3.4) leads to the conservation of mean energy under $\tau$ (but not the mean of functions of energy, such as its variance):

(3.5) Theorem. Let $T$ map each $\mathscr{B}\left(\mathscr{H}_{\eta}\right)$ and $\mathscr{L}$ into itself, and commute with permutations. Then the mean energy is invariant under $\tau$.

Proof. Let $\varrho \in \mathscr{B}(\mathscr{H})_{1}$ be such that

$$
\operatorname{Tr}(\varrho H)=\lim _{M \rightarrow \infty} \operatorname{Tr}\left(\varrho H_{M}\right)=\mathscr{E}<\infty .
$$

We note that

$$
\mathfrak{h}_{M}=H_{M} \otimes 1 \ldots \otimes 1+\ldots+1 \otimes \ldots \otimes 1 \otimes H_{M}
$$


Then by the symmetry of $T$

$$
\operatorname{Tr}\left(H_{M} \tau \varrho\right)=n^{-1} \operatorname{Tr}_{1 \ldots n}\left\{\mathfrak{h}_{M} T(\varrho \otimes \ldots \otimes \varrho)\right\} .
$$

This is a finite-dimensional trace and it can be evaluated in any basis, e.g. in a basis of eigenvectors of $\mathfrak{h}_{M}$. Then it involves only the block diagonal terms of $\varrho \otimes \ldots \otimes \varrho$, which are in $\mathscr{B}(\mathscr{H} \eta), \eta=0, \ldots, n M$. On each of these subspaces, $\mathfrak{h}_{M}$ is $\eta \cdot 1_{\mathscr{H}_{\eta}}$, and so commutes with $T$. Hence

$$
\operatorname{Tr}\left(H_{M} \tau \varrho\right)=n^{-1} \operatorname{Tr}_{1 \ldots n}\left(T\left(\mathfrak{h}_{M} \varrho \otimes \ldots \otimes \varrho\right)\right)=n^{-1} \operatorname{Tr}_{1 \ldots n}\left(\mathfrak{h}_{M} \varrho \otimes \ldots \otimes \varrho\right)
$$

as $T$ is trace-preserving

$$
=\operatorname{Tr}\left(H_{M} \varrho\right) .
$$

Hence $\lim _{M \rightarrow \infty} \operatorname{Tr}\left(H_{M} \tau \varrho\right)=\mathscr{E}$, so $\tau \varrho$ has finite mean energy, $\mathscr{E}$ the same value as $\varrho$.

(3.6) Remark. It has been pointed out by the referee that it is not enough to suppose that $T$ commutes with the time-evolution $A \mapsto e^{i \hbar t} A e^{-i \hbar t}$ of density matrices $A \in \mathscr{B}(\mathscr{H})_{1}$ : the mean energy fails to be conserved in general unless $T$ maps $\mathscr{B}\left(\mathscr{H}_{\eta}\right)$ and $\mathscr{L}$ to themselves. As a counterexample in two dimensions, let $\mathfrak{h}=\left(\begin{array}{ll}1 & 0 \\ 0 & 0\end{array}\right)$ and $T\left(\begin{array}{ll}a & b \\ b^{*} & c\end{array}\right)=\left(\begin{array}{ll}c & 0 \\ 0 & a\end{array}\right)$. Then $T$ commutes with $[\cdot, \mathfrak{h}]$, but does not leave the diagonal blocks invariant. Average energy is not invariant under $T$. Physically, such transformations $T$ are "too stochastic" and do not lead to the canonical ensemble.

\section{Weak * Convergence}

(4.1) The set of all states of $\mathscr{B}(\mathscr{K})$, not necessarily normal ones, is $w^{*}$-compact. The sequence $\left\{\tau^{m} \varrho\right\}_{m=0,1, \ldots}$ therefore has a $w^{*}$-convergent subnet $\left\{\varrho_{\alpha}\right\}_{\alpha \in I}$. If $\varrho$ has mean energy $\mathscr{E}$, then by (3.5)

$$
\operatorname{Tr}\left(\varrho_{\alpha} H\right)=\mathscr{E} \text { for } \alpha \in I .
$$

(4.3) The entropy of a state $\varrho \in \mathscr{B}(\mathscr{H})_{1}$ of finite mean energy is finite and $\leqq$ the entropy of the Gibbs state of the same energy. Since the entropy is non-decreasing under $\tau, S\left(\tau^{m} \varrho\right)$ converges as $m \rightarrow \infty$, and $S\left(\varrho_{\alpha}\right)$ converges to the same limit as $\alpha \rightarrow \infty$.

(4.4) Lemma. Let $\varrho_{\infty}=w^{*} \lim _{\alpha \rightarrow \infty} \varrho_{\alpha}$. Let $P_{j}=\varrho_{\infty}\left(E_{j}\right), j=0,1,2, \ldots$. Then $\lim _{j \rightarrow \infty} P_{j}=1$.

(4.5) Remark. This is tantamount to showing that $\varrho_{\infty}$ is normal.

(4.6) Proof. Let $p_{j}=P_{j}-P_{j-1}, j=0,1,2, \ldots$ and $p_{j}^{\alpha}=\operatorname{Tr} \varrho_{\alpha}\left(E_{j}-E_{j-1}\right)$. Then $\mathscr{E}=\operatorname{Tr}\left(\varrho_{\alpha} H\right)=\sum_{j} j p_{j}^{\alpha}$, and $p_{j}=\lim _{\alpha} p_{j}^{\alpha}$. Hence $p_{j}$ obeys the conditions of $[1,(3.15)]$, and so $\sum_{0}^{\infty} p_{j}=\lim _{j \rightarrow \infty}^{j} P_{j}=1$.

(4.7) It does not seem easy to prove that $\sum j p_{j}=\mathscr{E}$ unless, of course, $k=\operatorname{dim} \mathscr{K}<\infty$. This might indicate that, for certain initial states, energy can escape up the energy ladder, say, by "heat solitons". But since for any $M, \sum_{1}^{M} j p_{j}^{\alpha} \leqq \mathscr{E}$, we have $\sum_{1}^{M} j p_{j} \leqq \mathscr{E}$. Hence $\lim _{M \rightarrow \infty} \varrho_{\infty}\left(H_{M}\right)=\sum^{\infty} j p_{j} \leqq \mathscr{E}$ and the limit state $\varrho_{\infty}$ has finite mean energy $\leqq \mathscr{E}$. 
(4.8) We now give an estimate for the entropy in the tail of a state.

(4.9) Lemma. Let $H$ be as in (3.1), and $\varrho$ be a positive operator of trace class such that $\operatorname{Tr} \varrho=q$ and $\operatorname{Tr}(\varrho H) \leqq \mathscr{E}$. Then $-\operatorname{Tr}(\varrho \log \varrho)=O(q \log q)$ as $q \rightarrow 0$.

(4.10) Proof. The largest value of $-\operatorname{Tr} \varrho \log \varrho$, subject to the conditions $\operatorname{Tr}(\varrho H) \leqq \mathscr{E}, \operatorname{Tr} \varrho=q$ is achieved at the Gibbs-like operator $\varrho$, diagonal in a basis provided by the eigenvectors of $H$. Then the problem reduces to the classical case: maximize

$$
s=-\sum_{0} m(j) p_{j} \log p_{j}
$$

among sequences of non-negative numbers $\left\{p_{j}\right\}$ obeying the constraints

$$
\sum_{0} m(j) p_{j}=q, \quad \sum_{1} m(j) j p_{j} \leqq \mathscr{E}
$$

When the multiplicity $m(j)$ is 1 for all $j$, then Lemma 3.19 of [1] shows that

$$
s \leqq-2 q \log q+q(1+\log \mathscr{E})=O(q \log q) .
$$

The same method also works when $m(j) \leqq \kappa$. So we have proved the lemma when the index $r$ of (3.1) is zero. We proceed by induction on $r$. Suppose the lemma is true for all sequences $\left\{p_{j}\right\}$ obeying (3.3) with $m(j) \leqq \kappa j^{r-1}, j=1,2, \ldots$. Now let $\left\{p_{j}\right\}$ satisfy (4.11) with $m(j) \leqq \kappa j^{r}$. Write $\left\{p_{j}\right\}$ together with repetitions for multiplicity as the union of sequences $\left\{p_{j}^{(\alpha)}\right\}, \alpha=1,2, \ldots$ defined by

$$
p_{j}^{(\alpha)}= \begin{cases}p_{j} & \text { if } j \geqq \alpha, \\ 0 & \text { otherwise } .\end{cases}
$$

In the sequence $\left\{p_{j}^{(\alpha)}\right\}_{j=0,1, \ldots}$ we repeat $p_{j}^{(\alpha)}$ with multiplicity $m(\alpha, j)$ which might be 0 or as large as $m(j) / j \leqq \kappa j^{r-1}$. It is possible to do this so that $m(j)=\sum_{\alpha} m(\alpha, j)$. Define

$$
\begin{gathered}
q^{(\alpha)}=\sum_{j} m(\alpha, j) p_{j}^{(\alpha)} \\
\mathscr{E}^{(\alpha)}=\sum_{j} m(\alpha, j) j p_{j}^{(\alpha)} \leqq \mathscr{E} \\
s^{(\alpha)}=-\sum_{j} m(\alpha, j) p_{j}^{(\alpha)} \log p_{j}^{(\alpha)}
\end{gathered}
$$

Then $\sum_{\alpha} q^{(\alpha)}=q, \sum_{\alpha} \mathscr{E}^{(\alpha)} \leqq \mathscr{E}, \sum_{\alpha} s^{(\alpha)}=s$.

Now, the induction hypothesis implies that $s^{(\alpha)}=O\left(-q^{(\alpha)} \log q^{(\alpha)}\right)$ uniformly in $\alpha$. Also, the condition (4.12) implies $\sum \alpha q^{(\alpha)} \leqq \mathscr{E}$ :

$$
\begin{aligned}
\sum \alpha q^{(\alpha)} & =\sum_{\alpha} \sum_{i} \alpha m(\alpha, j) p_{j}^{(\alpha)} \leqq \sum_{\alpha} \sum_{j} j m(\alpha, j) p_{j}^{(\alpha)} \\
& \leqq \sum_{j} j \sum_{\alpha} m(\alpha, j) p_{j}^{(\alpha)} \leqq \sum_{j} j m(j) p_{j}=\mathscr{E}
\end{aligned}
$$

Thus $\left\{q^{(\alpha)}\right\}$ itself obeys the conditions of [1, Lemma 3.19], namely $\sum_{\alpha} q^{(\alpha)}=q$, $\sum \alpha q^{(\alpha)} \leqq \mathscr{E}$. So by $[1,(3.19)]$ :

$$
-\operatorname{Tr} \varrho \log \varrho \leqq s=0\left(\sum_{\alpha}-q^{(\alpha)} \log q^{(\alpha)}\right)=O(-q \log q)
$$


The main point is that $s \rightarrow 0$ as $q \rightarrow 0$. This result gives an extension of the classical theory [1] to the case with multiplicity $m(j)$ as in (3.1).

\section{Convergence to a Gibbs State}

(5.1) Suppose now that $T$ maps $\mathscr{L}$ to itself and each $\mathscr{B}\left(\mathscr{H}_{\eta}\right)$ to itself, and is ergodic on each $\mathscr{B}\left(\mathscr{H}_{\eta}\right)$. Let $\sigma_{m}=\tau^{m} \varrho \otimes \ldots \otimes \tau^{m} \varrho$ and let $\sigma_{m}(\eta)$ be the diagonal block matrix obtained from $\sigma_{m}$ by restricting to $\mathscr{H}_{\eta}$. Then, as in Theorem (2.5), we see that the component of $\sigma_{m}(\eta)$ orthogonal [in the sense of $\mathscr{B}(\mathscr{H})_{2}$ ] to multiples of the identity $1_{\mathscr{\ell}}$, converges to 0 as $m \rightarrow \infty$. In particular, the off-diagonal elements converge to 0 . This does not (yet) show that $\sigma_{m}(\eta)$ converges, as we have not controlled the trace. But along the convergent subnet $\varrho_{\alpha}$ we also get convergence of $\sigma_{\alpha}$ and of $\sigma_{\alpha}(\eta)$ : this must converge to a multiple of $1_{\mathscr{H}_{\eta}}$. To see clearly why this implies that $\varrho_{\infty}$ is diagonal in the energy basis, first take $n=2$. Write, in Dirac notation

$$
\varrho=\sum \varrho_{i j}^{\mu \nu}|\mu i\rangle\langle v j|,
$$

where $i, j$ are energy labels and $1 \leqq \mu \leqq m(i), 1 \leqq v \leqq m(j) ; \mu, v$ label the multiple states of energy $i, j$, respectively. Then $\sigma=\varrho \otimes \varrho$ has the off-diagonal terms

$$
\varrho_{i j}^{\mu \nu} \varrho_{i^{\prime} j^{\prime} \nu^{\prime}}^{\mu^{\prime}}|\mu i\rangle\left|\mu^{\prime} i^{\prime}\right\rangle\langle v j|\left\langle v^{\prime} j^{\prime}\right|,
$$

including the case $i \neq j$ or $\mu \neq v$ where $i^{\prime}=j, i=j^{\prime}, \mu^{\prime}=v, \mu=v^{\prime}$. Thus the coefficient $\varrho_{i j}^{\mu \nu} \varrho_{j i}^{\nu \mu}=\left|\varrho_{i j}^{\mu \nu}\right|^{2}$ converges to 0 as $m \rightarrow \infty$. This is the general off-diagonal element of $\varrho$. Thus $\varrho_{\infty}$ is diagonal in the energy basis.

If $n>2$ we note at least one diagonal element $\varrho_{k k}^{\mu \mu}$ does not converge to zero, by (4.4). Then if $(n-2) k+i+j=\eta$, the off-diagonal element of $\sigma(\eta)$,

$$
\varrho_{k k}^{\mu \mu} \ldots \varrho_{k k}^{\mu \mu} \varrho_{i j}^{\lambda v} \varrho_{j i}^{\nu \lambda}=\left|\varrho_{k k}^{\mu \mu}\right|^{n-2}\left|\varrho_{i j}^{\lambda \nu}\right|^{2},
$$

converges to zero for any $i, \lambda \neq j, v$; then $\varrho_{i j}^{\lambda v} \rightarrow 0$. Thus $\varrho_{\infty}$ is diagonal in the energy basis. The argument now reduces to the classical case [1]: in order for $\sigma_{\infty}=\varrho_{\infty} \otimes \ldots \otimes \varrho_{\infty}$ to be a multiple of the identity on each $H_{\eta}, \varrho_{\infty}$ being diagonal, we obtain the result: $\varrho_{\infty}$ is a Gibbs state, $\varrho_{\beta}$. From (4.7), its energy is $\leqq \mathscr{E}$. To be precise, we have shown that $\varrho_{\infty}$ coincides with $\varrho_{\beta}$ as a state on $\bigcup_{j} \mathscr{B}\left(E_{j} \mathscr{K}\right)$. Recalling that $\left\{E_{j}\right\}$ is the spectral resolution of $H$, we have for any $j$ and $A \in \mathscr{B}(\mathscr{K})$, $\varrho_{\infty}(A)=\varrho_{\infty}\left(E_{j} A E_{j}\right)+\varrho_{\infty}\left(\left(1-E_{j}\right) A E_{j}\right)+\varrho_{\infty}\left(E_{j} A\left(1-E_{j}\right)\right)+\varrho_{\infty}\left(\left(1-E_{j}\right) A\left(1-E_{j}\right)\right)$.

By Schwarz' inequality for states,

$$
\left|\varrho_{\infty}\left(1-E_{j}\right) A E_{j}\right| \leqq\left[\varrho_{\infty}\left(1-E_{j}\right)\right]^{1 / 2}\left[\varrho_{\infty}\left(E_{j} A^{*} A E_{j}\right)\right]^{1 / 2},
$$

and by (4.4), $\varrho_{\infty}\left(1-E_{j}\right) \rightarrow 0$ as $j \rightarrow \infty$, the other factor being bounded. Similarly, the other terms converge to 0 as $j \rightarrow \infty$. But $\varrho_{\infty}\left(E_{j} A E_{j}\right)=\varrho_{\beta}\left(E_{j} A E_{j}\right)$, and this converges to $\varrho_{\beta}(A)$ as $j \rightarrow \infty$, as $\varrho_{\beta}$ is normal. Hence $\varrho_{\infty}(A)=\varrho_{\beta}(A)$ for all $A \in \mathscr{B}(\mathscr{K})$.

(5.2) The same argument shows that any other $w^{*}$ convergent subnet $\left\{\varrho_{\beta}\right\}_{\beta \in J}$ of $\left\{\tau^{m} \varrho\right\}$ converges to a Gibbs state of energy $\leqq \mathscr{E}$, but (so far), it could be different from $\varrho_{\infty}$. We show they are the same by showing they have the same entropy, namely $\lim _{m} S\left(\tau^{m} \varrho\right)$. 
(5.3) Theorem. Under the above conditions, $S\left(\varrho_{\alpha}\right) \rightarrow S\left(\varrho_{\infty}\right), \alpha \rightarrow \infty$.

Proof. Choose $\varepsilon>0$. Write $\varrho_{\alpha}=E_{j} \varrho_{\alpha} E_{j}+A, A=\varrho_{\alpha}-E_{j} \varrho_{\alpha} E_{j} \geqq 0$ and

$$
\begin{aligned}
q & =\operatorname{Tr} A=\sum_{k=j}^{\infty} \operatorname{Tr}\left(E_{k+1}-E_{k}\right) \varrho_{\alpha} \leqq j^{-1} \sum_{k=j}^{\infty} \operatorname{Tr} k\left(E_{k+1}-E_{k}\right) \varrho_{\alpha} \\
& =j^{-1} \operatorname{Tr}\left(H \varrho_{\alpha}\right)=j^{-1} \mathscr{E} .
\end{aligned}
$$

Choose $j_{0}$ large enough so that $q$ is small enough so that, by (4.9), $S(A)<\varepsilon$ for all $\alpha$ and all $j \geqq j_{0}$. Then, by the subadditivity of the entropy [7],

$$
S\left(\varrho_{\alpha}\right) \leqq S\left(E_{j} \varrho_{\alpha} E_{j}\right)+S(A) \leqq S\left(E_{j} \varrho_{\alpha} E_{j}\right)+\varepsilon
$$

for all $\alpha$ and all $j \geqq j_{0}$. Since $E_{j} \varrho_{\alpha} E_{j}$ (j fixed) has finite rank, $S$ is continuous on this subspace. Taking limits of $(5.5)$ gives for $j \geqq j_{0}$ :

$$
S=\lim _{\alpha} S\left(\varrho_{\alpha}\right) \leqq \lim _{\alpha} S\left(E_{j} \varrho_{\alpha} E_{j}\right)+\varepsilon=S\left(E_{j} \varrho_{\infty} E_{j}\right)+\varepsilon .
$$

Taking the limit $j \rightarrow \infty$ gives $[8$, Appendix $] s \leqq S\left(\varrho_{\infty}\right)+\varepsilon$. Since this is true for every $\varepsilon>0$, we get $s \leqq S\left(\varrho_{\infty}\right)$. Now let $j$ be so large that

$$
S\left(\varrho_{\infty}\right) \leqq S\left(E_{j} \varrho_{\infty} E_{j}\right)+\frac{\varepsilon}{2} .
$$

This is possible [8, Appendix $]$.

For this $j$ choose $\alpha_{0}$ so large that for all larger $\alpha$,

Then

$$
S\left(E_{j} \varrho_{\alpha} E_{j}\right) \geqq S\left(E_{j} \varrho_{\infty} E_{j}\right)-\frac{\varepsilon}{2} .
$$

$$
S\left(\varrho_{\infty}\right) \leqq S\left(E_{j} \varrho_{\infty} E_{j}\right)+\frac{\varepsilon}{2} \leqq S\left(E_{j} \varrho_{\alpha} E_{j}\right)+\varepsilon \leqq S\left(\varrho_{\alpha}\right)+\varepsilon
$$

for all larger $\alpha$,

$$
\leqq s+\varepsilon
$$

Since this is true for every $\varepsilon>0$, we have $S\left(\varrho_{\infty}\right) \leqq s$. This gives $S\left(\varrho_{\infty}\right)=S$.

(5.8) We can now put together the results.

Theorem. Let $H$ be a self-adjoint operator on $\mathscr{K}$ with spectrum $0,1,2, \ldots$, and the finite multiplicity $m(j)$ of eigenvalue $j$ obeys $m(j) \leqq \kappa j^{r}, j=1,2, \ldots$. Let $\mathscr{H}=\mathscr{K} \otimes \ldots \otimes \mathscr{K}$, and let $T$ be a symmetry-preserving doubly stochastic map on $\mathscr{B}(\mathscr{H})_{1}, T$ mapping $\mathscr{L}$ and each $\mathscr{B}\left(\mathscr{H}_{\eta}\right)$ to itself and ergodic on each energy shell. Let $\tau$ be the corresponding Boltzmann map. Let $\varrho$ be any density matrix on $\mathscr{K}$ with finite mean energy $\mathscr{E}$.

Then $\tau^{m} \varrho$ converges as $m \rightarrow \infty$ in trace norm to a Gibbs state $\varrho_{\infty}=e^{-\beta H} / \operatorname{Tr} e^{-\beta H}$ of energy $\leqq \mathscr{E}$, as $m \rightarrow \infty$.

Proof. Any convergent subnet of $\left\{\tau^{m} \varrho\right\}$ converges $w^{*}$ to a Gibbs state (Sect. 5.1). All such limit states have the same entropy (Sect. 5.3) and are therefore the same. 
Therefore, $\left\{\tau^{m} \varrho\right\}$ converges in the $w^{*}$ topology to a Gibbs state. Its energy is $\leqq \mathscr{E}$, by Sect. 4.7. The convergence in trace-norm follows from

$$
\begin{aligned}
\left\|\tau^{m} \varrho-\varrho_{\infty}\right\|_{1} \leqq & \left\|\tau^{m} \varrho-E_{j} \tau^{m} \varrho E_{j}\right\|_{1} \\
& +\left\|E_{j} \varrho_{\infty} E_{j}-E_{j} \tau^{m} \varrho E_{j}\right\|_{1}+\left\|\varrho_{\infty}-E_{j} \varrho_{\infty} E_{j}\right\|_{1}
\end{aligned}
$$

and (5.4), using that $\tau^{m} \varrho \rightarrow \varrho_{\infty}$ when restricted to the finite-dimensional space $E_{j} \mathscr{K}$.

(5.9) If $T$ is not ergodic on the energy shells, but is ergodic when restricted to a smaller slice conserving two numbers (e.g. energy and particle number), we prove convergence to a grand canonical ensemble in a similar way.

(5.10) If $\operatorname{dim} \mathscr{K}<\infty$, then $\operatorname{Tr}(H \varrho)$ is continuous, and so $\varrho_{\infty}$ has mean energy $\mathscr{E}$. Then $\lim \tau^{m} \varrho$ is the same state for all $\varrho$ with mean energy $\mathscr{E}$.

Acknowledgements. I thank D. Ghikas for discussions about related problems, and the referee for criticism leading to a clearer version of the paper.

\section{References}

1. Streater, R.F.: Convergence of the iterated Boltzmann map (to appear in Publ. R.I.M.S., 1984)

2. Dixmier, J.: Les algèbres d'operateurs sur l'espaces hilbertiennes. Paris: Gauthier-Villars 1969

3. Manuceau, J. et al.: Entropy and normal states. Commun. Math. Phys. 27, 327 (1972)

4. Alberti, P.M., Uhlmann, A.: Stochasticity and partial order. Dordrecht: Reidel 1982

5. Alicki, R., Messer, J.: Non-linear quantum dynamic semigroups for many-body open systems. J. State Phys. 32, 299 (1983)

6. Ruelle, D.: Statistical mechanics. New York: Benjamin 1969

7. Lanford, O.E., Robinson, D.W.: Mean entropy of states in quantum-statistical mechanics. J. Math. Phys. 9, 1120 (1968)

8. Lieb, E.H., Ruskai, M.B.: Proof of the strong subadditivity of quantum-mechanical entropy. J. Math. Phys. 14, 1938 (1973) (Appendix by B. Simon)

Communicated by J. L. Lebowitz

Received September 29, 1983; in revised form June 14, 1984 
